TAIWANESE JOURNAL OF MATHEMATICS

Vol. 13, No. 6A, pp. 1695-1710, December 2009

This paper is available online at http://www.tjm.nsysu.edu.tw/

\title{
PROPER CLARKE EPIDERIVATIVE IN SET-VALUED OPTIMIZATION
}

\author{
C. S. Lalitha and R. Arora* \\ Dedicated to Professor Boris S. Mordukhovich in honor of his 60th birthday.
}

\begin{abstract}
Using the concept of Clarke tangent cone, a new notion of proper Clarke epiderivative for a set-valued map is introduced. Its nature and certain properties are investigated. Finally necessary and sufficient optimality conditions for a constrained set-valued optimization problem have been established in terms of proper Clarke epiderivative.
\end{abstract}

\section{INTRODUCTION}

The derivative of a map at a point has got its implication analytically as well as geometrically. The notion of derivatives for set-valued maps has been defined with the help of tangent cones. Aubin [1] introduced the notion of derivatives in terms of tangent cones for set-valued maps using the contingent cone. This derivative called contingent derivative, is defined as the set-valued map whose graph coincides with the contingent cone to the graph of the set-valued map. However prior to the work of Aubin [1] the notion of coderivative has been introduced for set-valued maps in terms of the basic normal cone to their graphs by Mordukhovich [20]. This concept of derivative is conceptually different from the tangential derivative due to the absence of duality between tangent and normal cones in nonconvex setting. However for the smooth and convex maps the two notions are equivalent.

In the past two decades a lot of research has been done to improvise the notion of derivative in terms of tangent cones. Using contingent derivative Corley [6] derived necessary and sufficient optimality conditions for weak efficient solutions whereas Luc [19] derived these conditions for local efficient solutions. Also Corley [6]

Received December 12, 2008.

2000 Mathematics Subject Classification: 54C60, 90C26, 90C30.

Key words and phrases: Set-valued optimization, Epiderivative, Proper minimizers, Tangent cone, Semilocal convexity.

The author is thankful to CSIR, India for the grant provided to support this research.

*Corresponding author. 
observed that the necessary and sufficient optimality conditions do not unify under the standard assumptions. So, derivatives involving epigraph of set-valued maps termed as epiderivatives have been considered in literature. In this direction Jahn and Rauh [14] introduced the concept of contingent epiderivative relating epigraph of the derivative with the contingent cone.

Chen and Jahn [5] introduced generalized contingent epiderivative in terms of minimizers of projection of the contingent cone to epigraph of a set-valued map. Jahn and Khan [12] introduced the notion of weak and proper contingent epiderivative in terms of weak and proper minimizers of projection sets respectively. Jahn and Khan [13] established various forms of optimality conditions for a set-valued optimization problem in terms of proper and weak contingent epiderivatives. For more details one can refer to the book by Jahn [11].

Chen [4] introduced the notion of tangent epiderivative (also termed Clarke epiderivative in [15]) of a set-valued map in terms of Clarke tangent cone where epiderivative is a single valued map. Lalitha, Dutta and Govil [15] obtained optimality conditions using this notion Clarke epiderivative for weak mnimizers. Recently Lalitha and Arora [16] introduced the notion of weak Clarke epiderivative in terms of weak minimizers of Clarke tangent cone. Bigi and Castellani [3] proposed a general definition of K-epiderivative and employed it to develop a general scheme for necessary optimality conditions in set-valued optimization problems.

Weak efficiency generalizes efficiency whereas proper efficiency refines efficiency by removing certain undesirable aspects of efficiency. The most important feature of proper efficiency is to eliminate unstable situations and to obtain some robustness of the solution, for instance, in situations where boundedness of the trade off rates is required or to establish optimality with respect to a larger ordering cone. Apart from the stability aspect it has been observed that there are efficient solutions, which fail to get characterized by a scalar optimization problem even if the decision set is convex. Apart from the work of Jahn and Khan [12,13] various authors have dealt with different kinds of proper efficient solutions in set-valued optimization and discussed optimality conditions (see Liu and Gong [17], Song et al. [24], Gong et al. [9], Gong [8]).

Since Clarke tangent cone is convex and proper efficiency is a refinement of the concept of efficiency, it is significant to study the notion of an epiderivative in terms of proper minimizer considering the Clarke tangent cone. The main aim of the paper is to introduce this notion, termed as proper Clarke epiderivative. The paper also deals with issues related to the nature and properties of proper Clarke epiderivative and establishes an optimality criterion for a constrained set-valued optimization problem.

The paper is organized as follows. Section 2 presents some basic definitions and results used in the paper. In Section 3 the notion of proper Clarke epiderivative is 
introduced and its nature, characterization and properties are investigated. Section 4 deals with both Fritz John and Kuhn Tucker type necessary optimality criteria as well as sufficient optimality criterion under a weakened form of cone convexity assumption namely semilocal cone convexity. In Section 5 certain observations are made regarding the relation of proper Clarke epiderivative with the existing generalized notions of epiderivatives. Finally some concluding remarks are made in the end.

\section{Preliminaries}

Let $Y$ be a real normed space partially ordered by a closed convex pointed cone $K$ in $Y$ and let $Y^{*}$ be the dual space of $Y$. Let $B$ be a nonempty set in $Y$ and $0_{Y}$ be the zero of $Y$. We first recall two well known notions of minimality of sets.

An element $\bar{y} \in B$ is said to be a minimizer of $B$ if $(B-\bar{y}) \cap(-K)=\left\{0_{Y}\right\}$ and a proper minimizer of $B$ if there exists a convex cone $K_{1} \subset Y$ such that $K \backslash\left\{0_{Y}\right\} \subset \operatorname{int} K_{1}$ and $\bar{y}$ is a minimizer of $B$ with respect to the cone $K_{1}$.

The set of all minimizers and proper minimizers of $B$ is denoted by $\operatorname{Min}(B, K)$, and $\operatorname{PMin}(B, K)$ respectively. It is obvious that $\operatorname{PMin}(B, K) \subseteq \operatorname{Min}(B, K)$.

We denote the closure of $B$ and interior of $B$ by $\operatorname{cl} B$ and int $B$ respectively. Also, cone $B$ denotes cone generated by $B$, that is, cone $B:=\{\lambda b \mid b \in B, \lambda \geq 0\}$. A nonempty convex subset $C$ of the convex cone $K$ is called a base of $K$ if $K=$ cone $C$ and $0_{Y} \notin \operatorname{cl} C$. The dual cone $K^{*}$ and the strict dual $K^{\#}$ of $K$ are defined as

$$
\begin{aligned}
K^{*} & :=\left\{l \in Y^{*} \mid\langle l, k\rangle \geq 0, \forall k \in K\right\} ; \\
K^{\#} & :=\left\{l \in Y^{*} \mid\langle l, k\rangle>0, \forall k \in K \backslash\left\{0_{Y}\right\}\right\} .
\end{aligned}
$$

The Clarke tangent cone to $B$ at $\bar{y} \in B$ is defined as

$$
\begin{array}{r}
T(B, \bar{y}):=\left\{y \in Y \mid \forall \bar{y}_{n} \rightarrow \bar{y}, \bar{y}_{n} \in B, t_{n}>0, t_{n} \rightarrow \infty, \exists y_{n} \in B\right. \\
\text { such that } \left.y_{n} \rightarrow \bar{y} \text { and } t_{n}\left(y_{n}-\bar{y}_{n}\right) \rightarrow y\right\} .
\end{array}
$$

Equivalently it is defined as

$$
\begin{array}{r}
T(B, \bar{y}):=\left\{y \in Y \mid \forall \bar{y}_{n} \rightarrow \bar{y}, \bar{y}_{n} \in B, t_{n} \downarrow 0, \exists y_{n} \rightarrow y\right. \\
\text { with } \left.\bar{y}_{n}+t_{n} y_{n} \in B \forall n\right\} .
\end{array}
$$

It can be seen that $T(B, \bar{y})$ is a closed convex cone.

Let $X$ be another real normed space and let $F: X \rightarrow 2^{Y}$ be a set-valued map. Throughout we assume that $K$ is a pointed closed convex cone in $Y$. The domain, graph and epigraph of $F$ are defined as

$$
\operatorname{dom} F:=\{x \in X \mid F(x) \neq \phi\}
$$




$$
\begin{aligned}
\text { graph } F & :=\{(x, y) \in X \times Y \mid x \in \operatorname{dom} F, y \in F(x)\} ; \\
\text { epi } F & :=\{(x, y) \in X \times Y \mid x \in \operatorname{dom} F, y \in F(x)+K\} .
\end{aligned}
$$

The profile map $P F: X \rightarrow 2^{Y}$ of $F$ is defined as

$$
P F(x)=F(x)+K \text {. }
$$

We now consider the notion of Clarke epiderivative for set-valued map where the epiderivative is a single valued map (see Chen [4], Lalitha, Dutta and Govil [15]).

Given $(\bar{x}, \bar{y}) \in$ graph $F$, a single valued map $D_{e} F(\bar{x}, \bar{y}): X \rightarrow Y$ whose epigraph is the Clarke tangent cone to epi $F$ at $(\bar{x}, \bar{y})$, that is,

$$
\text { epi } D_{e} F(\bar{x}, \bar{y})=T(\operatorname{epi} F,(\bar{x}, \bar{y}))
$$

is called the Clarke epiderivative of $F$ at $(\bar{x}, \bar{y})$.

In practice there are numerous set-valued maps for which Clarke epiderivative does not exist. For example consider a set-valued map $F: R \rightarrow 2^{R^{2}}$ defined by

$$
F(x)=\left\{(-y, y) \in R^{2} \mid 0 \leq y \leq x+1\right\}
$$

for every $x \in R$ and $K=R_{+}^{2}$. Here the epigraph is nonconvex and for $\bar{x}=0, \bar{y}=$ $(0,0)$

$$
T(\operatorname{epi} F,(\bar{x}, \bar{y}))=\left\{\left(x, y_{1}, y_{2}\right) \in R^{3} \mid y_{1} \leq 0, y_{1}+y_{2} \geq 0\right\}+\{0\} \times R_{+}^{2} .
$$

Clearly the Clarke epiderivative at $(\bar{x}, \bar{y})$ does not exist for any $x$ in $\operatorname{dom} F$.

Occurrence of such situations motivated the introduction of the concept of generalized tangent epiderivative by Chen [4]. In this case the projection of the Clarke tangent cone to epi $F$ at $(\bar{x}, \bar{y})$ on the image space is taken at a point and the value of the generalized Clarke epiderivative at a point is given by the minimizer of the projection set at that point. A set-valued map $D_{g} F(\bar{x}, \bar{y}): X \rightarrow 2^{Y}$ is said to be the generalized tangent epiderivative of $F$ at $(\bar{x}, \bar{y})$ if

$$
D_{g} F(\bar{x}, \bar{y})(x)=\operatorname{Min}(G(x), K)
$$

where

$$
G(x):=\{y \in Y \mid(x, y) \in T(\operatorname{epi} F,(\bar{x}, \bar{y}))\} .
$$

It can be easily observed that $G(x)$ is a closed convex set and $G(x)=G(x)+K$.

In the example considered above the generalized tangent epiderivative exists for each $x$ and is given by

$$
D_{g} F(\bar{x}, \bar{y})(x)=\left\{\left(y_{1}, y_{2}\right) \in R^{2} \mid y_{1}+y_{2}=0, y_{1} \leq 0\right\} .
$$




\section{Proper Clarke Epiderivative}

Although the concept of efficiency is quite relevant in the study of optimality of a set-valued optimization problem it has certain limitations namely the lack of stability conditions or inability to obtain a scalar characterization for efficient solutions. In order to avoid such undesirable efficient solutions, the notion of proper efficiency has been introduced by various authors. In the same spirit, we now introduce the concept of proper Clarke epiderivative in terms of proper minimizers of the projection set.

Definition 3.1. Given $(\bar{x}, \bar{y}) \in \operatorname{graph} F$, a set-valued map $D_{p} F(\bar{x}, \bar{y}): X \rightarrow 2^{Y}$ is said to be the proper Clarke epiderivative of $F$ at $(\bar{x}, \bar{y})$ if

$$
D_{p} F(\bar{x}, \bar{y})(x)=\operatorname{PMin}(G(x), K) .
$$

Based on the relation between proper minimizers and minimizers, it is obvious that

$$
D_{p} F(\bar{x}, \bar{y})(x) \subseteq D_{g} F(\bar{x}, \bar{y})(x)
$$

for all $x$ in $X$.

In the example considered above $D_{p} F(\bar{x}, \bar{y})(x)$ exists and coincides with $D_{g}$ $F(\bar{x}, \bar{y})(x)$ for all $x$ in $\operatorname{dom} F$. We now provide an example where proper Clarke epiderivative exists and is a proper subset of the generalized tangent epiderivative.

Example 3.1. Let $F: R \rightarrow 2^{R^{2}}$ be a set-valued map defined by

$$
F(x)= \begin{cases}R^{2} & \text { if } x \geq 0 \\ \left\{\left(y_{1}, y_{2}\right) \in R^{2} \mid y_{1}^{2}+y_{2}^{2} \geq x^{2}, y_{1} \geq 0, y_{2} \geq 0\right\} & \text { if } x<0\end{cases}
$$

and $K=R_{+}^{2}$. Clearly epi $F$ is a nonconvex set. For $\bar{x}=0, \bar{y}=(0,0)$

$T($ epi $F,(\bar{x}, \bar{y}))=\left\{\left(x, y_{1}, y_{2}\right) \in R^{3} \mid x \geq 0, y_{1}^{2}+y_{2}^{2} \leq x^{2}, y_{1} \leq 0, y_{2} \leq 0\right\}+\{0\} \times R_{+}^{2}$

and $G: R \rightarrow 2^{R^{2}}$ is given as

$$
G(x)= \begin{cases}\left\{\left(y_{1}, y_{2}\right) \in R^{2} \mid y_{1}^{2}+y_{2}^{2} \leq x^{2}, y_{1} \leq 0, y_{2} \leq 0\right\}+R_{+}^{2} & \text { if } x \geq 0 \\ \phi & \text { if } x<0\end{cases}
$$

Hence

$$
D_{p} F(\bar{x}, \bar{y})(x)= \begin{cases}\left\{\left(y_{1}, y_{2}\right) \in R^{2} \mid y_{1}^{2}+y_{2}^{2}=x^{2}, y_{1}<0, y_{2}<0\right\} & \text { if } x>0 \\ \{(0,0)\} & \text { if } x=0 \\ \phi & \text { if } x<0\end{cases}
$$


which is a proper subset of

$D_{g} F(\bar{x}, \bar{y})(x)= \begin{cases}\left\{\left(y_{1}, y_{2}\right) \in R^{2} \mid y_{1}^{2}+y_{2}^{2}=x^{2}, y_{1} \leq 0, y_{2} \leq 0\right\} & \text { if } x \geq 0 \\ \phi & \text { if } x<0 .\end{cases}$

Observe that for $x=0$, both the epiderivatives coincide.

Next we have an existence theorem for the proper Clarke epiderivative in finite dimensional case. For this we first we recall the following definitions.

Definition 3.2. $[14,18]$. Let $B \subseteq Y$ and $\bar{y} \in Y$.

(i) The set $B \cap(\bar{y}-K)$ is said to be a $K$-lower section of $B$ at $\bar{y}$.

(ii) The set $B$ is said to be minorized if there exists $y \in Y$ such that $B \subseteq\{y\}+K$.

(iii) The cone $K$ is said to be Daniell if any decreasing sequence in $Y$ having a lower bound converges to its infimum.

In view of the existence theorems for the efficient points in Luc [18], the following conclusions can be made when $Y$ is a finite dimensional space.

Theorem 3.1. Let $Y$ be a finite dimensional space $R^{m}$ and $F: X \rightarrow 2^{Y}$ be a set-valued map. For $(\bar{x}, \bar{y}) \in$ graph $F, D_{p} F(\bar{x}, \bar{y})$ exists if either of the following conditions hold:

(i) $G(x)$ has a nonempty minorized $K$-lower section for every $x \in \operatorname{dom} G$;

(ii) $G(x)$ has a nonempty bounded $K$-lower section for every $x \in \operatorname{dom} G$.

Proof. Since a pointed closed convex cone in a finite dimensional space is Daniell (see [10]) and $G(x)+K$ is a closed convex set in $Y$, the proof follows on using Corollary 3.8 (i) and (iii) and Corollary 3.16 (chapter 2) of Luc [18].

Remark 3.1. The conditions imposed on $G(x)$ in the above theorem cannot be relaxed as illustrated by the following example. If $F: R \rightarrow 2^{R^{2}}$ is a set-valued map defined by $F(x)=\{(y, 0) \mid y \leq 0\}, \forall x \in R$ then epi $F$ is a convex set and $G(x)=\left\{\left(y_{1}, y_{2}\right) \mid y_{2} \geq 0\right\}$. Observe that $D_{p} F(\bar{x}, \bar{y})(x)$ does not exist for any $x$ in $R$ as none of the $K$-lower sections of $G(x)$ is bounded or minorized.

Apart from the conditions under which the set of efficient points of a set is nonvoid, another important aspect in the theory of decision making is about the existence of an efficient alternative which is smaller than a given alternative with respect to the same ordering cone. This is the domination property, which has been studied widely by many researchers. The set $B$ is said to satisfy domination property if $B \subseteq \operatorname{Min}(B, K)+K$. For the set of proper minimizers, we now introduce a refined form of this domination property termed as the strong domination property. 
Definition 3.3. The set $B \subseteq Y$ is said to satisfy the strong domination property if

$$
B \subseteq \operatorname{clPMin}(B, K)+K .
$$

Remark 3.2. It is easy to observe that

$$
\operatorname{cl} \operatorname{PMin}(B, K)+K \subseteq \operatorname{cl}(\operatorname{PMin}(B, K)+K) .
$$

Hence if strong domination property holds then

$$
B \subseteq \operatorname{cl}(\operatorname{PMin}(B, K)+K) .
$$

In the definition, the proper Clarke epiderivative is given in terms of projection set (see (1)) but in the following two theorems we give some direct relations of proper Clarke epiderivative and Clarke tangent cone.

Theorem 3.2. For $(\bar{x}, \bar{y}) \in$ graph $F$ if $D_{p} F(\bar{x}, \bar{y})$ exists and strong domination property holds for $G(x)$ for $x \in X$ then

$$
\operatorname{cl} \text { epi } D_{p} F(\bar{x}, \bar{y})=T(\operatorname{epi} F,(\bar{x}, \bar{y})) .
$$

Moreover, if $D_{e} F(\bar{x}, \bar{y})$ exists then

$$
\operatorname{cl} \text { epi } D_{p} F(\bar{x}, \bar{y})=\operatorname{epi} D_{e} F(\bar{x}, \bar{y}) .
$$

Proof. Let $(x, y) \in \operatorname{cl}$ epi $D_{p} F(\bar{x}, \bar{y})$ which implies that there exists a sequence $\left\{\left(x_{n}, y_{n}\right)\right\}$ in epi $D_{p} F(\bar{x}, \bar{y})$ such that $\left(x_{n}, y_{n}\right) \rightarrow(x, y)$. As $y_{n} \in D_{p} F(\bar{x}, \bar{y})\left(x_{n}\right)+$ $K \subseteq G\left(x_{n}\right)+K=G\left(x_{n}\right)$, therefore $\left(x_{n}, y_{n}\right) \in T(\operatorname{epi} F,(\bar{x}, \bar{y}))$ which being closed implies $(x, y) \in T($ epi $F,(\bar{x}, \bar{y}))$. Conversely for $(x, y) \in T($ epi $F,(\bar{x}, \bar{y}))$ we have $y \in G(x)$. Since strong domination property holds for $G(x)$, by Remark 3.2 it follows that $y \in \operatorname{cl}(\operatorname{PMin}(G(x), K)+K)$ and hence there exists a sequence $\left\{y_{n}\right\}$ in $\operatorname{PMin}(G(x), K)+K$ such that $y_{n} \rightarrow y$. As $y_{n} \in D_{p} F(\bar{x}, \bar{y})(x)+K$, therefore $\left(x, y_{n}\right) \in \operatorname{epi} D_{p} F(\bar{x}, \bar{y})$, that is, $(x, y) \in \operatorname{clepi} D_{p} F(\bar{x}, \bar{y})$.

Theorem 3.3. If $D_{p} F(\bar{x}, \bar{y})$ exists for $(\bar{x}, \bar{y}) \in \operatorname{graph} F$, and if $D_{p} F(X):=$ $\cup\left\{D_{p} F(\bar{x}, \bar{y})(x) \mid x \in \operatorname{dom} G\right\}$ and $F(X):=\cup\{F(x) \mid x \in \operatorname{dom} F\}$ then

$$
D_{p} F(\bar{x}, \bar{y})(X) \subseteq T(F(X)+K, \bar{y}) .
$$

Proof. If $T(F(X)+K, \bar{y})=Y$, there is nothing to prove. For $T(F(X)+$ $K, \bar{y}) \neq Y$, let $x \in \operatorname{dom} G$ and $y \in D_{p} F(\bar{x}, \bar{y})(x) \subseteq G(x)$. Clearly $(x, y) \in$ $T($ epi $F,(\bar{x}, \bar{y}))$ and hence by the definition of Clarke tangent cone, for every $\left(\bar{x}_{n}, \bar{y}_{n}\right) \rightarrow(\bar{x}, \bar{y})$ with $\left(\bar{x}_{n}, \bar{y}_{n}\right) \in$ epi $F$ and $t_{n} \downarrow 0$ there exists $\left(x_{n}, y_{n}\right) \rightarrow(x, y)$ such that $\left(\bar{x}_{n}, \bar{y}_{n}\right)+t_{n}\left(x_{n}, y_{n}\right) \in$ epi $F$. This implies $\bar{y}_{n}+t_{n} y_{n} \in F\left(\bar{x}_{n}+t_{n} x_{n}\right)+K \subseteq$ $F(X)+K$ for every positive integer $n$ or equivalently $y \in T(F(X)+K, \bar{y})$. 

exist.

The following theorem relates $D_{p} F(\bar{x}, \bar{y}), D_{g} F(\bar{x}, \bar{y})$ and $D_{e} F(\bar{x}, \bar{y})$ if they

Theorem 3.4. For $(\bar{x}, \bar{y}) \in \operatorname{graph} F$ if $D_{p} F(\bar{x}, \bar{y})$ and $D_{e} F(\bar{x}, \bar{y})$ both exist then for each $x \in \operatorname{dom} G, D_{p} F(\bar{x}, \bar{y})(x)$ and $D_{g} F(\bar{x}, \bar{y})(x)$ belong to the boundary of $D_{e} F(\bar{x}, \bar{y})(x)+K$.

Proof. As $D_{e} F(\bar{x}, \bar{y})(x)+K$ is closed and $D_{p} F(\bar{x}, \bar{y})(x) \subseteq D_{g} F(\bar{x}, \bar{y})(x)$ for every $x \in \operatorname{dom} G$, therefore the result follows by proving that $D_{g} F(\bar{x}, \bar{y})(x)$ does not belong to the interior of $D_{e} F(\bar{x}, \bar{y})(x)+K$, for each $x$ in $\operatorname{dom} G$. For $y \in D_{g} F(\bar{x}, \bar{y})(x)$ we have $(x, y) \in \operatorname{epi} D_{e} F(\bar{x}, \bar{y})$, that is, $y \in D_{e} F(\bar{x}, \bar{y})(x)+K$. If $y \in \operatorname{int}\left(D_{e} F(\bar{x}, \bar{y})(x)+K\right)$ for some $x \in \operatorname{dom} G \subseteq X$, then there exist $\varepsilon>$ 0 and a corresponding open sphere $B_{\varepsilon}(y)$ of radius $\varepsilon$, such that $y \in B_{\varepsilon}(y) \subseteq$ $D_{e} F(\bar{x}, \bar{y})(x)+K$. As $B_{\varepsilon}(y)=y+B_{\varepsilon}(0)$, therefore for any $k \in K \backslash\left\{0_{Y}\right\}$, there exists $\eta>0$, sufficiently small such that $-\eta k \in B_{\varepsilon}(0)$, which implies that $y-\eta k \in B_{\varepsilon}(y) \subseteq D_{e} F(\bar{x}, \bar{y})(x)+K$. Equivalently $(x, y-\eta k) \in \operatorname{epi} D_{e} F(\bar{x}, \bar{y})=$ $T($ epi $F,(\bar{x}, \bar{y}))$ which further implies $-\eta k \in G(x)-y$. Also as $k \in K \backslash\left\{0_{Y}\right\}$ and $\eta>0$ we have $-\eta k \in-K \backslash\left\{0_{Y}\right\}$ and thus $-\eta k \in(G(x)-y) \cap\left(-K \backslash\left\{0_{Y}\right\}\right)$ which contradicts the fact that $y \in \operatorname{Min}(G(x), K)$.

Set-valued optimization problems like any other vector optimization problems lack completeness in preference orders. To overcome difficulties caused by incompleteness of the orders, techniques that convert vector problems into appropriate scalar problems are widely applied.

Scalarization of proper minimizers is quite well known for different forms of proper efficient points. For proper minimzers of the form considered in this paper refer to Theorems 3.1.2, 3.4.1 and 3.4.2 in Sawargi, Nakayama and Tanino [23], Theorem 2.1 in Gong [8] and Theorem 2.11 (Chapter 4) in Luc [18]. Likewise we have the following characterization for proper Clarke epiderivative.

Theorem 3.5. For $(\bar{x}, \bar{y}) \in \operatorname{graph} F$ if $D_{p} F(\bar{x}, \bar{y})$ exists and $K$ has a compact base then

$$
D_{p} F(\bar{x}, \bar{y})(x)=\cup\left\{\langle k, G(x)\rangle^{-} \mid k \in K^{\#}\right\}
$$

for each $x \in \operatorname{dom} G$ where $\langle k, G(x)\rangle^{-}:=\{y \in G(x) \mid\langle k, y\rangle \leq\langle k, G(x)\rangle\}$ and $\langle k, G(x)\rangle:=\{\langle k, y\rangle \mid y \in G(x)\}$.

Proof. Follows on the lines of Proposition 4.2 of Jahn and Khan [12] as Clarke tangent is convex.

In Example 3.1, it can be seen that for $k=\left(k_{1}, k_{2}\right) \in K^{\#}$ and $x \in \operatorname{dom} G$ the set $\langle k, G(x)\rangle^{-}=\left\{\left(y_{1}, y_{2}\right) \mid y_{1}=-t k_{1}, y_{2}=-t k_{2}, t=x / \sqrt{k_{1}^{2}+k_{2}^{2}}\right\}$. Since 
$k \in K^{\#}$ it follows that $\cup\left\{\langle k, G(x)\rangle^{-} \mid k \in K^{\#}\right\}=\left\{\left(y_{1}, y_{2}\right) \in R^{2} \mid y_{1}^{2}+y_{2}^{2}=\right.$ $\left.x^{2}, y_{1}<0, y_{2}<0\right\}$ which coincides with $D_{p} F(\bar{x}, \bar{y})(x)$ for each $x$ in $\operatorname{dom} G$.

Remark 3.3. For $(\bar{x}, \bar{y}) \in \operatorname{graph} F$ if $D_{p} F(\bar{x}, \bar{y})(x)$ exists for all $x$ in $\operatorname{dom} G$, then it can be observed that it is strictly positive homogeneous, that is, for all $x \in X$ and $\alpha>0, D_{p} F(\bar{x}, \bar{y})(\alpha x)=\alpha D_{p} F(\bar{x}, \bar{y})(x)$. This follows as $G(\alpha x)=\alpha G(x)$ and $\operatorname{PMin}(\alpha B, K)=\alpha \operatorname{PMin}(B, K)$ for $\alpha>0$.

\section{Optimality Conditions}

In this section we deal with optimality conditions for a constrained set-valued optimization problem in terms of proper Clarke epiderivative. We consider the following set-valued optimization problem

$$
\begin{array}{ll}
(\mathrm{VP}) & \operatorname{Min} F(x) \\
& \text { subject to } H(x) \cap(-D) \neq \phi
\end{array}
$$

where $H: X \rightarrow 2^{Z}$ is a set-valued map and $D$ is a closed convex pointed cone in real normed space $Z$.

The feasible region is given by the set $S:=\{x \in X \mid H(x) \cap(-D) \neq \phi\}$. The image set of $S$ under $F$ is given by $F(S)=\cup\{F(x) \mid x \in S\}$.

Definition 4.1. A point $(\bar{x}, \bar{y}, \bar{z})$ is said to be a proper minimizer of (VP) if $\bar{x} \in S, \bar{y} \in F(\bar{x}) \cap \operatorname{PMin}(F(S), K)$ and $\bar{z} \in H(\bar{x}) \cap(-D)$.

To establish Kuhn Tucker type necessary and sufficient optimality criteria we require certain convexity assumptions of the set-valued maps. In this regard we consider the notion of semilocal cone convexity, a generalization of cone convexity.

We first recall the following notion of local star shapedness introduced by Ewing [7]. A set $A$ in $X$ is said to be locally star shaped at $\bar{x} \in A$ if for any $x \in A$, there exists a positive real number $a(x, \bar{x}) \leq 1$ such that $(1-\lambda) \bar{x}+\lambda x \in A$ for $0<\lambda \leq a(x, \bar{x})$. The set $A$ is said to be locally star shaped if it is locally star shaped at each of its points.

The set $A=\left\{(x, y) \in R^{2}\left|x^{2} \leq\right| y \mid\right\} \cup\left\{(x, y) \in R^{2} \mid y=0\right\}$ is locally star shaped at origin but is not convex in any neighborhood containing origin.

Let $A \subseteq X$ be a locally star shaped set at $\bar{x} \in A$ and $F: A \rightarrow 2^{Y}$ be a setvalued map such that $\operatorname{dom} F=A$. The map $F$ is said to be semilocally $K$-convex at $\bar{x}$ if for every $x \in A, y \in F(x)$ and $\bar{y} \in F(\bar{x})$ there exists a positive real number $d((x, y),(\bar{x}, \bar{y})) \leq a(x, \bar{x})$ such that

$$
(1-\lambda) \bar{y}+\lambda y \in F((1-\lambda) \bar{x}+\lambda x)+K \text { for } 0<\lambda \leq d((x, y),(\bar{x}, \bar{y})) .
$$


$F$ is said to be semilocally $K$-convex on $A$ if $F$ is semilocally $K$-convex at each $x \in A$.

Remark 4.1. If $A$ is locally star shaped and $d((x, y),(\bar{x}, \bar{y}))=a(x, \bar{x})=1$ for every $x, \bar{x} \in A, y \in F(x)$ and $\bar{y} \in F(\bar{x})$ then clearly $A$ is a convex set and every semilocally $K$-convex set-valued map on $A$ is $K$-convex on $A$.

On using standard arguments the following lemma can be easily established.

Lemma 4.1. A set-valued map $F: A \rightarrow 2^{Y}$ defined on a locally star shaped set $A$ is semilocally $K$-convex if and only if epi $F$ is a locally star shaped set.

It can be observed that semilocally $K$-convex set-valued map is a generalization of locally $K$-convex set-valued map considered in [13]. We recall that a set-valued map $F: X \rightarrow 2^{Y}$ is locally $K$-convex at $(\bar{x}, \bar{y}) \in$ graph $F$ if there exists a neighborhood $N$ of $(\bar{x}, \bar{y})$ such that the set $\operatorname{cl}(N \cap$ epi $F)$ is convex.

We recall that a set-valued map $F: X \rightarrow 2^{Y}$ is said to be closed at $x \in X$ if for sequences $\left\{x_{n}\right\}$ in $X$ and $\left\{y_{n}\right\}$ in $Y$ with $y_{n} \in F\left(x_{n}\right) \subseteq Y, x_{n} \rightarrow x$ and $y_{n} \rightarrow y$ we have $y \in F(x)$. F is said to be closed on $X$ if $F$ is closed at every $x \in X$.

Since a set-valued map is closed if and only if its graph is a closed set we can conclude that the epigraph of a set-valued map $F$ is closed if the profile map $P F$ is closed.

The following theorem gives a representation of a semilocal cone convex setvalued map defined on $X$ in terms of proper Clarke epiderivative.

Theorem 4.1. Suppose that $F: X \rightarrow 2^{Y}$ is a semilocally $K$-convex set-valued map on $X$ such that the following hold:

(a) $D_{p} F(\bar{x}, \bar{y})$ exists for $(\bar{x}, \bar{y}) \in \operatorname{graph} F$;

(b) $\operatorname{dom} G=X$;

(c) strong domination property holds for $G(x)$ for every $x \in X$;

(d) profile map $P D_{p} F(\bar{x}, \bar{y})$ is closed;

then

$$
F(x)-\{\bar{y}\} \subseteq D_{p} F(\bar{x}, \bar{y})(x-\bar{x})+K
$$

for every $x \in X$.

Proof. In order to prove the above result we need to prove that for $(x, y) \in$ graph $F$ the implication $y-\bar{y} \in D_{p} F(\bar{x}, \bar{y})(x-\bar{x})+K$, that is, $(x-\bar{x}, y-$ $\bar{y}) \in$ epi $D_{p} F(\bar{x}, \bar{y})$ holds. Since the profile map $P D_{p} F(\bar{x}, \bar{y})$ is closed we have 
epi $D_{p} F(\bar{x}, \bar{y})=\operatorname{cl}$ epi $D_{p} F(\bar{x}, \bar{y})$ and since strong domination property holds, from Theorem 3.2, we have cl epi $D_{p} F(\bar{x}, \bar{y})=T($ epi $F,(\bar{x}, \bar{y}))$. Therefore it is enough to prove that $(x-\bar{x}, y-\bar{y}) \in T(\operatorname{epi} F,(\bar{x}, \bar{y}))$. Let $\left(\bar{x}_{n}, \bar{y}_{n}\right) \rightarrow(\bar{x}, \bar{y})$ with $\left(\bar{x}_{n}, \bar{y}_{n}\right) \in$ epi $F$ and $t_{n} \rightarrow \infty$ with $t_{n}>0$. Since epi $F$ is a locally star shaped set there exists a positive real number $d\left((x, y),\left(\bar{x}_{n}, \bar{y}_{n}\right)\right) \leq 1$ such that $\left((1-\lambda) \bar{x}_{n}+\lambda x,(1-\right.$ $\left.\lambda) \bar{y}_{n}+\lambda y\right) \in$ epi $F$ for $0 \leq \lambda \leq d\left((x, y),\left(\bar{x}_{n}, \bar{y}_{n}\right)\right)$. Without loss of generality we can assume that $0<1 / t_{n} \leq d\left((x, y),\left(\bar{x}_{n}, \bar{y}_{n}\right)\right)$ for each $n$. Define $x_{n}=$ $\left(1-1 / t_{n}\right) \bar{x}_{n}+\left(1 / t_{n}\right) x$ and $y_{n}=\left(1-1 / t_{n}\right) \bar{y}_{n}+\left(1 / t_{n}\right) y$ for each $n$. Hence for each $n$ we have $x_{n} \in X, y_{n} \in F\left(x_{n}\right)+K,\left\{x_{n}\right\} \rightarrow \bar{x}$ and $\left\{y_{n}\right\} \rightarrow \bar{y}$. This implies $\left(x_{n}, y_{n}\right) \in \operatorname{epi} F,\left(x_{n}, y_{n}\right) \rightarrow(\bar{x}, \bar{y})$ and $t_{n}\left\{\left(x_{n}, y_{n}\right)-\left(\bar{x}_{n}, \bar{y}_{n}\right)\right\} \rightarrow(x-\bar{x}, y-\bar{y})$.

In the following theorems we use the set-valued map $(F, H): X \rightarrow 2^{Y \times Z}$ defined as $(F, H)(x):=F(x) \times H(x)$, for every $x \in X$. For $(\bar{x}, \bar{y}, \bar{z}) \in$ graph $(F, H)$ define

$$
G^{\prime}(x):=\{(y, z) \in Y \times Z \mid(x, y, z) \in T(\operatorname{epi}(F, H),(\bar{x}, \bar{y}, \bar{z}))\} .
$$

Theorem 4.2. If $(\bar{x}, \bar{y}, \bar{z})$ is a proper minimizer of $(V P)$ such that the following conditions hold:

(i) strong domination property holds for $G^{\prime}(x)$ for every $x \in X$;

(ii) $G^{\prime}(0)$ is pointed;

then there exists $(\varphi, \psi) \in K^{*} \times D^{*} \backslash\left\{\left(0_{Y}, 0_{Z}\right)\right\}$ such that for all $(y, z) \in$ $D_{p}(F, H)(\bar{x}, \bar{y}, \bar{z})(X)$

(i) $\varphi(y)+\psi(z) \geq 0$

(ii) $\psi(\bar{z})=0$;

where $0_{Z}$ is the zero of $Z$.

Proof. The proof follows from Theorem 3.1 in [4] as a proper minimzer is a minimizer, strong domination property implies domination property and $D_{p}(F, H)(\bar{x}, \bar{y}, \bar{z})(X) \subseteq D_{g}(F, H)(\bar{x}, \bar{y}, \bar{z})(X)$.

Next we have the Kuhn Tucker type necessary optimality criteria for (VP) under Slater's type constraint qualification and semilocal cone convexity conditions. We say the problem (VP) satisfies the generalized Slater's constraint qualification if there exists $x^{\prime} \in X$ such that $H\left(x^{\prime}\right) \cap(-\operatorname{int} D) \neq \phi$.

Theorem 4.3. If $(\bar{x}, \bar{y}, \bar{z})$ is a proper minimizer of $(V P)$ such that the following conditions hold:

(a) strong domination property holds for $G^{\prime}(x)$ for every $x \in X$; 
(b) $G^{\prime}(0)$ is pointed;

(c) $\operatorname{dom} G^{\prime}=X$;

(d) $F$ is semilocally $K$-convex on $X$ and $H$ is semilocally $D$-convex on $X$;

(e) profile map $P D_{p}(F, H)(\bar{x}, \bar{y}, \bar{z})$ is closed;

$(f)$ Slater's constraint qualification holds;

then there exist $\varphi \in K^{*} \backslash\left\{0_{Y}\right\}$ and $\psi \in D^{*}$ such that for all $(y, z) \in D_{p}(F, H)$ $(\bar{x}, \bar{y}, \bar{z})(X)$ conditions (i) and (ii) of Theorem 4.2 hold.

Proof. As $(\bar{x}, \bar{y}, \bar{z})$ is a proper minimizer of (VP) therefore by Theorem 4.2, there exists $(\varphi, \psi) \in K^{*} \times D^{*} \backslash\left\{\left(0_{Y}, 0_{Z}\right)\right\}$ such that $\psi(\bar{z})=0$ and $\varphi(y)+\psi(z)$ $\geq 0$ for each $(y, z)$ in $D_{p}(F, H)(\bar{x}, \bar{y}, \bar{z})(X)$. In view of Theorem 4.1 for any $(y, z) \in F(S) \times H(S)$ we have $(y, z)-(\bar{y}, \bar{z}) \in D_{p}(F, H)(\bar{x}, \bar{y}, \bar{z})(x-\bar{x})+K \times D$. Since $\varphi(k) \geq 0$ for every $k \in K$ and $\psi(d) \geq 0$ for every $d \in D$, it follows that $\varphi(y-\bar{y})+\psi(z-\bar{z}) \geq 0$, that is $\varphi(y-\bar{y})+\psi(z) \geq 0$. If $\varphi=0_{Y}$ then $\psi \neq 0_{Z}$ and hence for all $z \in H(S)$, we get $\psi(z) \geq 0$. As the generalized Slater's constraint qualification is satisfied there exists $x^{\prime} \in X$ such that $H\left(x^{\prime}\right) \cap(-$ int $D) \neq \phi$ which implies that there exists $z^{\prime} \in H\left(x^{\prime}\right) \cap(-\operatorname{int} D)$. Since $z^{\prime} \in-\operatorname{int} D$ it follows that $\psi\left(z^{\prime}\right)<0$ which is a contradiction.

In order to establish the sufficiency optimality criteria, Chen [4] assumed the cone convexity of the objective function and the set-valued map involved in the constraints while dealing with the generalized tangent epiderivatives. However in case of proper Clarke epiderivative we impose semilocal cone convexity assumptions on the maps.

Theorem 4.4. Suppose that for $\bar{x} \in S, \bar{y} \in F(\bar{x}), \bar{z} \in H(\bar{x}) \cap(-D)$ there exist $\varphi \in K_{1}^{\#} \subseteq K^{*} \backslash\left\{0_{Y}\right\}$ and $\psi \in D^{*}$ such that for all $(y, z) \in D_{p}(F, H)(\bar{x}, \bar{y}, \bar{z})(X)$ conditions (i) and (ii) of Theorem 4.2 hold where $K_{1}$ is a pointed convex cone satisfying $K \backslash\left\{0_{Y}\right\} \subset \operatorname{int} K_{1}$. Moreover assume that the following conditions hold:

(a) strong domination property holds for $G^{\prime}(x)$ for every $x \in X$;

(b) $\operatorname{dom} G^{\prime}=X$;

(c) $F$ is semilocally $K$-convex on $X$ and $H$ is semilocally $D$-convex on $X$;

(d) profile map $P D_{p}(F, H)(\bar{x}, \bar{y}, \bar{z})$ is closed;

then $(\bar{x}, \bar{y}, \bar{z})$ is a proper minimizer of $(V P)$.

Proof. In view of Theorem 4.1 for any $(y, z) \in F(S) \times H(S)$ we have $(y, z)$ $-(\bar{y}, \bar{z}) \in D_{p}(F, H)(\bar{x}, \bar{y}, \bar{z})(x-\bar{x})+K \times D$. Since $K \backslash\left\{0_{Y}\right\} \subset \operatorname{int} K_{1}$ it follows that $\varphi \in K^{*}$ and on using the hypothesis we have $\varphi(y-\bar{y})+\psi(z) \geq 0$ for any 
$(y, z) \in F(S) \times H(S)$. If $\bar{y} \notin \operatorname{PMin}(F(S), K)$ then for every pointed convex cone $K^{\prime}$ containing $K \backslash\left\{0_{Y}\right\}$ in its interior, $\bar{y} \notin \operatorname{Min}\left(F(S), K^{\prime}\right)$. In particular $\bar{y} \notin \operatorname{Min}\left(F(S), K_{1}\right)$, that is, there exists $\hat{x} \in S, \hat{y} \in F(\hat{x}), \hat{y} \neq \bar{y}$ such that $\varphi(\hat{y}-\bar{y})<0$. Since $\hat{x} \in S$ there exists $\hat{z} \in H(\hat{x}) \cap(-D)$ which satisfies the relation $\psi(\hat{z}) \leq 0$. Hence $\varphi(\hat{y}-\bar{y})+\psi(\hat{z})<0$ which is a contradiction.

\section{Relation with Contingent Epiderivatives}

In the following theorem we use the notion of a contingent (Bouligand tangent) cone to $B$ at $\bar{y} \in B$ defined as

$$
\begin{array}{r}
T_{c}(B, \bar{y}):=\left\{y \in Y \mid \exists y_{n} \rightarrow \bar{y}, y_{n} \in B, t_{n}>0, t_{n} \rightarrow \infty,\right. \\
\text { such that } \left.t_{n}\left(y_{n}-\bar{y}\right) \rightarrow y\right\} .
\end{array}
$$

Equivalently

$$
T_{c}(B, \bar{y}):=\left\{y \in Y \mid \exists y_{n} \rightarrow \bar{y}, y_{n} \in B, t_{n} \downarrow 0 \text { such that } \bar{y}+t_{n} y_{n} \in B\right\} .
$$

It can be seen that $T_{c}(B, \bar{y})$ is a closed cone, $T(B, \bar{y}) \subseteq T_{c}(B, \bar{y})$ and $T(B, \bar{y})=$ $T_{c}(B, \bar{y})=\mathrm{cl}$ cone $(B-\bar{y})$ if $B$ is a convex set.

We now study the relations between the notions of Clarke epiderivatives and the contingent epiderivatives considered by Jahn and Khan [12]. The epiderivatives in terms of the contingent cone are defined as the respective minimizers of the projection set $G_{c}(x)$ of the contingent cone on the image space given by the set

$$
G_{c}(x):=\left\{y \in Y \mid(x, y) \in T_{c}(\operatorname{epi} F,(\bar{x}, \bar{y}))\right\}, \forall x \in X .
$$

Based on the nature of the contingent cone, it can be seen that $G_{c}(x)$ is a closed set. Moreover $G(x) \subseteq G_{c}(x)$, for every $x \in \operatorname{dom} G$ and $G(x)=G_{c}(x)$ if $T_{c}($ epi $F,(\bar{x}, \bar{y}))$ is a convex set.

We now recall the definitions of the generalized contingent epiderivative and proper contingent epiderivative given by Jahn and Khan [12]. Let $F: X \rightarrow 2^{Y}$ be a set-valued map with $\bar{y} \in F(\bar{x})$ for $\bar{x} \in X$. A set-valued map $D_{g}^{c} F(\bar{x}, \bar{y}): X \rightarrow 2^{Y}$ defined by

$$
D_{g}^{c} F(\bar{x}, \bar{y})=\operatorname{Min}\left(G_{c}(x), K\right)
$$

is the generalized contingent epiderivative of $F$ at $(\bar{x}, \bar{y})$ and a set-valued map $D_{p}^{c} F(\bar{x}, \bar{y}): X \rightarrow 2^{Y}$ defined by

$$
D_{p}^{c} F(\bar{x}, \bar{y})=\operatorname{PMin}\left(G_{c}(x), K\right)
$$

is the proper contingent epiderivative of $F$ at $(\bar{x}, \bar{y})$.

We now have a set-valued map for which the proper and the generalized contingent epiderivatives do not exist whereas the proper Clarke epiderivative and the generalized tangent epiderivative exist. 
Example 5.1. For the set-valued map $F: R \rightarrow 2^{R^{2}}$ defined by $F(x)=$ $\{(x, 0) \mid x \leq 0\} \cup\{(0, x) \mid x \leq 0\}$ and $K=R_{+}^{2}$. In this case for $\bar{x}=0, \bar{y}=$ $(0,0), T_{c}($ epi $F,(\bar{x}, \bar{y}))=R \times\left(R^{2} \backslash \operatorname{int} R_{-}^{2}\right)$. Therefore $G_{c}(x): R \rightarrow R^{2}$ is given by $G_{c}(x)=R^{2} \backslash \operatorname{int} R_{-}^{2}$ and hence $D_{g}^{c} F(\bar{x}, \bar{y})(x)=D_{p}^{c} F(\bar{x}, \bar{y})(x)=\phi$. As $T(\operatorname{epi} F,(\bar{x}, \bar{y}))=R \times R_{+}^{2}$ we have $G(x)=R_{+}^{2}$ and hence $D_{g} F(\bar{x}, \bar{y})(x)=$ $D_{p} F(\bar{x}, \bar{y})(x)=\{(0,0)\}$, for every $x \in R$.

The proper contingent epiderivative even if it exists may differ from the proper Clarke epiderivative as illustrated by the following example.

Example 5.2. Let $F: R \rightarrow 2^{R^{2}}$ be a set-valued map defined by $F(x)=$ $\left\{\left(y_{1}, y_{2}\right) \in R^{2} \mid y_{2} \geq-y_{1}, y_{1} \leq 0\right\} \cup\left\{\left(y_{1}, y_{2}\right) \in R^{2} \mid y_{2} \geq-2 y_{1}, y_{1} \geq 0\right\}$ for all $x$ in $R$ and $K=R_{+}^{2}$. Here it can be seen that for $\bar{x}=0, \bar{y}=(0,0), D_{g}^{c} F(\bar{x}, \bar{y})(x)=$ $D_{p}^{c} F(\bar{x}, \bar{y})(x)=\left\{\left(y_{1}, y_{2}\right) \in R^{2} \mid y_{2}=-y_{1}, y_{1} \leq 0\right\} \cup\left\{\left(y_{1}, y_{2}\right) \in R^{2} \mid y_{2}=\right.$ $\left.-2 y_{1}, y_{1} \geq 0\right\}$ for all $x$ in $\operatorname{dom} G$ whereas $D_{g} F(\bar{x}, \bar{y})(x)=D_{p} F(\bar{x}, \bar{y})(x)=$ $\left\{\left(y_{1}, y_{2}\right) \in R^{2} \mid y_{2}=-y_{1}, y_{1} \geq 0\right\} \cup\left\{\left(y_{1}, y_{2}\right) \in R^{2} \mid y_{2}=-2 y_{1}, y_{1} \leq 0\right\}$ for all $x$ in $\operatorname{dom} G$.

If the proper and generalized contingent epiderivatives and corresponding Clarke epiderivatives exist for $(\bar{x}, \bar{y}) \in \operatorname{grah} F$, then for every $x \in \operatorname{dom} G$,

(i) $D_{p}^{c} F(\bar{x}, \bar{y})(x) \cap G(x) \subseteq D_{p} F(\bar{x}, \bar{y})(x) \subseteq D_{p}^{c} F(\bar{x}, \bar{y})(x)+K$;

(ii) $D_{g}^{c} F(\bar{x}, \bar{y})(x) \cap G(x) \subseteq D_{g} F(\bar{x}, \bar{y})(x) \subseteq D_{g}^{c} F(\bar{x}, \bar{y})(x)+K$;

(iii) $D_{p} F(\bar{x}, \bar{y})(x) \subseteq D_{g} F(\bar{x}, \bar{y})(x) \subseteq D_{g}^{c} F(\bar{x}, \bar{y})(x)+K$.

If the set-valued map is convex around the point $(\bar{x}, \bar{y}) \in$ graph $F$ then proper Clarke epiderivative coincides with contingent epiderivative as the Clarke tangent cone and contingent cone coincide at that point. This holds for instance for locally $K$-convex map.

\section{Conclusions}

The notion of proper Clarke epiderivative has been introduced in this paper and its nature has been studied. An optimality criterion has been established in terms of proper Clarke epiderivative. The study of this new notion is significant because it refines the existing class of generalized Clarke epiderivative. Moreover to obtain the Kuhn Tucker necessary optimality theorem and sufficiency criteria in terms of proper Clarke epiderivative the convexity assumptions taken are more general.

It would be further interesting to derive optimality conditions using variational principle and generalized differentiation tools (see [21, 22]) for set valued maps derived by Bao and Mordukhovich [2]. 


\section{REFERENCES}

1. J. P. Aubin, Contingent derivatives of set-valued maps and existence of solutions to nonlinear inclusions and differential inclusions, in: Mathematical analysis and applications, Part A, L. Nachbin, (ed.), 7a, Academic Press, New York-London, 1981, pp. 159-229.

2. T. Q. Bao and B. S. Mordukhovich, Variational principles for set-valued mappings with applications to multiobjective optimization, Cont. Cyber, 36 (2007), 531-562.

3. G. Bigi and M. Castellani, K-epiderivatives for set-valued functions and optimization, Math. Meth. Oper. Res., 55 (2002), 401-412.

4. L. Chen, Generalized tangent epiderivative and applications to set-valued map optimization, J. Nonlinear Convex Anal., 3 (2002), 303-313.

5. G. Y. Chen and J. Jahn, Optimality conditions for set-valued optimization problems, Math. Methods Oper. Res., 48 (1998), 187-200.

6. H. W. Corley, Optimality conditions for maximizations of set-valued functions, $J$. Optim. Theory Appl., 58 (1988), 1-10.

7. G. M. Ewing, Sufficient conditions for global minima of suitably convex functionals from variational and control theory, SIAM Review, 19 (1977), 202-220.

8. X. H. Gong, Optimality conditions for Henig and globally proper efficient solutions with ordering cone has empty interior, J. Math. Anal. Appl., 307 (2005), 12-31.

9. X. H. Gong, H. B. Dong and S. Y. Wang, Optimality conditions for proper efficient solutions of vector set-valued optimization, J. Math. Anal. Appl., 284 (2003), 332350 .

10. A. Gopfert, H. Riahi, C. Tammer and C. Zalinescu, Variational methods in partially ordered spaces, CMS Books in Mathematics, Springer-Verlag, New York, 2003.

11. J. Jahn, Vector Optimization, Theory, Applications, and Extensions, Springer-Verlag, Berlin, Heidelberg, 2004.

12. J. Jahn and A. A. Khan, Existence theorems and characterizations of generalized contingent epiderivatives, J. Nonl. Convex Anal., 3 (2002), 315-330.

13. J. Jahn and A. A. Khan, Generalized contingent epiderivatives in set-valued optimization: optimality conditions, Numer. Funct. Anal. Optim., 23 (2002), 807-831.

14. J. Jahn, and R. Rauh, Contingent epiderivatives and set-valued optimization, Math. Meth. Oper. Res., 46 (1997), 193-211.

15. C. S. Lalitha, J. Dutta and M. G. Govil, Optimality conditions in set-valued optimization, J. Aust. Math. Soc., 75 (2003), 1-11.

16. C. S. Lalitha and R. Arora, Weak Clarke epiderivative in set-valued optimization, $J$. Math. Anal. Appl., 342 (2008), 704-714.

17. W. Liu and X. H. Gong, Proper efficiency for vector set-valued optimization problems and vector variational inequalities, Math. Methods Oper. Res., 51 (2000), 443-457. 
18. D. T. Luc, Theory of vector optimization, Lecture Notes in Economics and Mathematical Systems, 319, Springer Verlag, 1989.

19. D. T. Luc, Contingent derivatives of set-valued maps and applications to vector optimization, Math. Prog., 50 (1991), 99-111.

20. B. S. Mordukhovich, Metric approximations and necessary optimality conditions for general classes of extremal problems, Soviet Math. Doklady, 22 (1980), 526-530.

21. B. S. Mordukhovich, Variational analysis and generalized differentiation, Vol. 1: Basic theory, Springer Verlag, Berlin et al., 2006.

22. B. S. Mordukhovich, Variational analysis and generalized differentiation, Vol. 2: Applications, Springer Verlag, Berlin et al., 2006.

23. Y. Sawaragi, H. Nakayama and T. Tanino, Theory of multiobjective optimization, Academic Press, Orlando, 1985.

24. J. Song, H. B. Dong and X. H. Gong, Proper efficiency in vector set-valued optimization problem, J. Nanchang Univ., 25 (2001), 122-130.

C. S. Lalitha

Department of Mathematics,

University of Delhi South Campus,

New Delhi - 110021,

India

E-mail: cslalitha@ rediffmail.com

R. Arora

Department of Mathematics,

University of Delhi,

Delhi 110007,

India

E-mail: ruchiiq@yahoo.co.in 